\title{
On the lines passing through two conjugates of a Salem number
}

\author{
BY ARTŪRAS DUBICKAS \\ Department of Mathematics and Informatics, Vilnius University, \\ Naugarduko 24, Vilnius LT-03225, Lithuania. \\ e-mail: arturas.dubickas@maf.vu.1t \\ AND CHRIS SMYTH \\ School of Mathematics and Maxwell Institute for Mathematical Sciences, \\ University of Edinburgh, James Clerk Maxwell Bld., King's Buildings, \\ Mayfield Road Edinburgh EH 93JZ, Scotland. \\ e-mail: c.smyth@ed.ac.uk
}

(Received 13 October 2006; revised 5 January 2007)

\begin{abstract}
We show that the number of distinct non-parallel lines passing through two conjugates of an algebraic number $\alpha$ of degree $d \geqslant 3$ is at most $\left[d^{2} / 2\right]-d+2$, its conjugates being in general position if this number is attained. If, for instance, $d \geqslant 4$ is even, then the conjugates of $\alpha \in \overline{\mathbb{Q}}$ of degree $d$ are in general position if and only if $\alpha$ has 2 real conjugates, $d-2$ complex conjugates, no three distinct conjugates of $\alpha$ lie on a line and any two lines that pass through two distinct conjugates of $\alpha$ are non-parallel, except for $d / 2-1$ lines parallel to the imaginary axis. Our main result asserts that the conjugates of any Salem number are in general position. We also ask two natural questions about conjugates of Pisot numbers which lead to the equation $\alpha_{1}+\alpha_{2}=\alpha_{3}+\alpha_{4}$ in distinct conjugates of a Pisot number. The Pisot number $\alpha_{1}=(1+\sqrt{3+2 \sqrt{5}}) / 2$ shows that this equation has such a solution.
\end{abstract}

\section{Introduction}

Recall that a Salem number $\alpha>1$ is an algebraic integer of degree $d \geqslant 4$ over the field of rational numbers $\mathbb{Q}$ whose conjugates, other than $\alpha$ itself, are $\alpha^{-1}$ and $d-2$ numbers of modulus 1 . The degree $d$ of a Salem number must be even. Setting $d=2 m+2$, where $m$ is a positive integer, one can write the full set of conjugates of a Salem number as $\alpha, \alpha^{-1}, e^{i \varphi_{1}}, e^{-i \varphi_{1}}, \ldots, e^{i \varphi_{m}}, e^{-i \varphi_{m}}$, where $0<\varphi_{1}<\cdots<\varphi_{m}<\pi$. In his book, Salem proved that the arguments $\varphi_{1}, \ldots, \varphi_{m}$ and $\pi$ are linearly independent over $\mathbb{Q}$ (see [5, p. 32]; the proof is attributed to Pisot). This implies that there is no non-trivial multiplicative relation between the conjugates of a Salem number, that is $\left(e^{i \varphi_{1}}\right)^{k_{1}}\left(e^{i \varphi_{2}}\right)^{k_{2}} \cdots\left(e^{i \varphi_{m}}\right)^{k_{m}} \neq 1$ if at least one of the integers $k_{1}, \ldots, k_{m}$ is nonzero.

In this paper, we prove the following:

THEOREM 1. No three distinct conjugates of a Salem number lie on a line. 
THEOREM 2. No two lines that pass through two distinct conjugates of a Salem number of degree $d$ are parallel, apart from the d/2-1 lines parallel to the imaginary axis passing through complex conjugate pairs.

The proofs of Theorems 1 and 2 will be given in Sections 3 and 4, respectively. In contrast to the above mentioned result of Salem, and unlike similar results for Pisot numbers [1] and [3] (see Section 5) whose proofs are based on just one "trick", the proofs of both these theorems are non-trivial.

In Section 5, we raise a few natural questions about conjugates of a Pisot number. However, we begin with the following problem: find the maximal number of distinct non-parallel lines passing through two conjugates of an algebraic number of degree $d$.

\section{The maximum number of lines passing through conjugate pairs}

Let $\alpha$ be an algebraic number of degree $d \geqslant 2$ over $\mathbb{Q}$. There are $d(d-1) / 2$ pairs of conjugates, so, in general, there are $d(d-1) / 2$ lines passing through each such pair. For $d=2$, there is indeed only one line. However, for $d \geqslant 3$, some of those lines can be parallel or even coincide. Suppose that $d=r+2 s$, where $r$ is the number of real conjugates of $\alpha$ and $2 s$ is the number of complex conjugates. If $r \geqslant 3$ then there are $r(r-1) / 2$ lines (among the $d(d-1) / 2)$ which coincide with the real axis. If $s \geqslant 2$ then $s$ lines passing through pairs of complex conjugate roots will be parallel to the imaginary axis. Hence, given any $d \geqslant 3$, it is quite natural to ask what is the maximum number $N$ such that there is an algebraic number of degree $d$ with $N$ distinct non-parallel lines passing through pairs of its conjugates.

In order to answer this question for any $d \geqslant 3$, we just need, by the above, to find the maximum of the expression

$$
D(r, 2 s):=d(d-1) / 2-\max \{r(r-1) / 2-1,0\}-\max \{s-1,0\},
$$

where $r$ and $s$ are two non-negative integers satisfying $d=r+2 s$. We claim that the maximum for $D(r, 2 s)$ is equal to

$$
\left[d^{2} / 2\right]-d+2
$$

where $[\ldots]$ stands for the integral part of a number.

Indeed, if $d \geqslant 4$ is even then, using $r+2 s=d$ and the fact that $r$ is even, we obtain that

$$
D(2, d / 2-1)=d(d-1) / 2-d / 2+2=d^{2} / 2-d+2=\left[d^{2} / 2\right]-d+2 .
$$

On the other hand, the numbers $D(0, d / 2)=d(d-1) / 2-d / 2+1=d^{2} / 2-d+1, D(d, 0)=1$ and

$$
\begin{aligned}
D(r, 2 s) & =d(d-1) / 2-r(r-1) / 2+1-s+1 \\
& =d(d-1) / 2-r(r-1) / 2-(d-r) / 2+2=d^{2} / 2-d+2+r-r^{2} / 2,
\end{aligned}
$$

where $4 \leqslant r \leqslant d-2$, are all strictly smaller than $d^{2} / 2-d+2$. This implies that, for every even $d \geqslant 4$, the number of distinct non-parallel lines passing through two conjugates of an algebraic number of degree $d$ is at most $\left[d^{2} / 2\right]-d+2$.

Similarly, for $d \geqslant 3$ odd, $D(r, 2 s)$ attains its maximum at $r=1$ and $s=(d-1) / 2$, giving

$$
D(1,(d-1) / 2)=\left(d^{2}-2 d+3\right) / 2=\left[d^{2} / 2\right]-d+2 .
$$

Furthermore, in both (even and odd) cases, the maximal number $\left[d^{2} / 2\right]-d+2$ is attained for $\alpha \in \overline{\mathbb{Q}}$ of degree $d$ precisely when no three conjugates of $\alpha$ lie on a line and no two lines 
passing through two distinct conjugates of $\alpha$ each are parallel, except for [(d-1)/2] lines (for $d \geqslant 5$ ) through complex conjugate pairs which are parallel to the imaginary axis. We then say that the conjugates of such an $\alpha$ are in general position.

In particular, if $d \geqslant 4$ is even, then the conjugates of $\alpha \in \overline{\mathbb{Q}}$ of degree $d$ are in general position if and only if $\alpha$ has 2 real conjugates, $d-2$ complex conjugates, no three distinct conjugates of $\alpha$ lie on a line, and any two lines that are passing through two distinct conjugates of $\alpha$ are non-parallel, except for $d / 2-1$ lines (when $d \geqslant 6$ ) parallel to the imaginary axis. Clearly, Theorems 1 and 2 combine to give the following corollary:

\section{COROLlaRY 3. The conjugates of any Salem number are in general position.}

In fact, for any $d \geqslant 3$, there are many algebraic numbers $\alpha$ of degree $d$ whose conjugates are in general position. For example, the conjugates of any non-totally real cubic algebraic number are in general position. It is an easy exercise to show that the conjugates of a quartic algebraic number $\alpha$ with two real conjugates $\alpha_{1}$ and $\alpha_{2}$ and two non-real conjugates $\alpha_{3}$ and $\overline{\alpha_{3}}$ are in general position except when $\alpha_{1}+\alpha_{2}=\alpha_{3}+\overline{\alpha_{3}}$, which means that the numbers $\alpha_{1}, \alpha_{3}, \alpha_{2}, \overline{\alpha_{3}}$ in the complex plane form a rhombus. We conclude this section with the following explicit example for each $d \geqslant 3$.

THEOREM 4. Let $f$ be an irreducible polynomial of degree $d \geqslant 3$ over $\mathbb{Q}$ having one real root for $d$ odd and two real roots for d even whose Galois group is isomorphic to the full symmetric group $S_{d}$. Then the roots of $f$ are in general position. In particular, the roots of $x^{d}-x-1$, where $d \geqslant 3$, are in general position.

Proof. It is well known (see for instance [2, p. 42]) that, for each $d \geqslant 2$, the polynomial $g(x):=x^{d}-x-1$ is irreducible and its Galois group $G$ is isomorphic to the full symmetric group $S_{d}$. We shall use this fact repeatedly in our argument.

Set $x_{d}:=d^{-1 /(d-1)}$. Clearly $g\left(x_{d}\right)<0$, the derivative of $g(x)$ is zero at $x= \pm x_{d}$ for $d$ odd and at $x_{d}$ for $d$ even. In the first case, $g\left(-x_{d}\right)<0$, so $g(x)$ has a unique real zero for each odd $d$. In the second case $g(x)$ has two real zeros for each even $d \geqslant 2$. This shows that the first claim of the theorem implies the second concerning the roots of the polynomial $x^{d}-x-1$.

Let $f$ be a polynomial satisfying the conditions of the theorem, and let $\alpha$ be one of the real roots of $f$. We denote the roots of $f$ by $\alpha_{1}, \ldots, \alpha_{d}$. In order to prove the first claim of the theorem we need to show that no three roots of $f$ lie on a line, and that no two lines passing through two roots of $f$ are parallel, except for those [ $(d-1) / 2]$ that for $d \geqslant 5$ are all parallel to the imaginary axis.

We remark first that none of the remaining $d(d-1) / 2-[(d-1) / 2]$ lines is parallel to the imaginary axis. Indeed, if the line going through $\alpha_{1}, \alpha_{2}$, where $\alpha_{1}$ is non-real and $\alpha_{2} \neq \alpha_{1}, \overline{\alpha_{1}}$, is parallel to the imaginary axis then $\alpha_{1}+\overline{\alpha_{1}}=\alpha_{2}+\overline{\alpha_{2}}$. Now, if $\alpha_{2}$ is real then $\alpha_{1}+\overline{\alpha_{1}}=2 \alpha_{2}$, which is impossible, by lemma 1 of [4]. Suppose that $\alpha_{2}$ is non-real. Then there is an automorphism $\sigma \in G$ which maps $\alpha_{2}$ to the real conjugate $\alpha$ and fixes each of the conjugates $\overline{\alpha_{2}}, \alpha_{1}$, and $\overline{\alpha_{1}}$. Then $\alpha_{1}+\overline{\alpha_{1}}=\alpha_{2}+\overline{\alpha_{2}}$ combined with $\alpha_{1}+\overline{\alpha_{1}}=\alpha+\overline{\alpha_{2}}$ implies that $\alpha=\alpha_{2}$, a contradiction.

Two lines passing through $\alpha_{1}, \alpha_{2}$ and $\alpha_{3}, \alpha_{4}$, where $\alpha_{1}, \alpha_{2}, \alpha_{3}$ are distinct, $\alpha_{4} \neq \alpha_{2}, \alpha_{3}$, but $\alpha_{4}$ can be equal to $\alpha_{1}$ (this corresponds to the case when $\alpha_{1}, \alpha_{2}, \alpha_{3}$ lie on a line), are parallel if there is a real constant $k$ such that $\alpha_{1}-\alpha_{2}=k\left(\alpha_{3}-\alpha_{4}\right)$. Evidently, then $\overline{\alpha_{1}}-\overline{\alpha_{2}}=k\left(\overline{\alpha_{3}}-\overline{\alpha_{4}}\right)$, 
so that

$$
\frac{\alpha_{1}-\alpha_{2}}{\overline{\alpha_{1}}-\overline{\alpha_{2}}}=\frac{\alpha_{3}-\alpha_{4}}{\overline{\alpha_{3}}-\overline{\alpha_{4}}} .
$$

We will show that this equation is impossible. It is clear that the algebraic numbers $\alpha_{1}, \ldots, \alpha_{4}, \overline{\alpha_{1}}, \ldots, \overline{\alpha_{4}}$ are all conjugate to $\alpha_{1}$. They are not necessarily all distinct, but $\alpha_{3} \notin\left\{\alpha_{1}, \alpha_{2}, \alpha_{4}\right\}$. So, in principle, $\alpha_{3}$ can be any of the numbers $\overline{\alpha_{1}}, \overline{\alpha_{2}}, \overline{\alpha_{3}}, \overline{\alpha_{4}}$.

Suppose that the list $\alpha_{1}, \ldots, \alpha_{4}, \overline{\alpha_{1}}, \ldots, \overline{\alpha_{4}}$, which we denote by $L$, contains at least one number, say, without loss of generality, $\alpha_{1}$ exactly once. Then $\alpha_{1}$ must be non-real and the list $L$ contains $\overline{\alpha_{1}}$ only once too. As above, there is an automorphism $\sigma \in G$ that transposes $\alpha_{1}$ with $\overline{\alpha_{1}}$ and fixes all other conjugates of $\alpha_{1}$. Setting $X:=\left(\alpha_{3}-\alpha_{4}\right) /\left(\overline{\alpha_{3}}-\overline{\alpha_{4}}\right)$, we obtain that $\alpha_{1}-\alpha_{2}=X\left(\overline{\alpha_{1}}-\overline{\alpha_{2}}\right)$ and $\overline{\alpha_{1}}-\alpha_{2}=X\left(\alpha_{1}-\overline{\alpha_{2}}\right)$. Subtracting one equation from the other we get $\left(\alpha_{1}-\overline{\alpha_{1}}\right)(1-X)=0$, so $X=1$. Hence $\alpha_{1}-\overline{\alpha_{1}}=\alpha_{2}-\overline{\alpha_{2}}$. Since $\left\{\alpha_{2}, \overline{\alpha_{2}}\right\} \neq\left\{\alpha_{1}, \overline{\alpha_{1}}\right\}$, by taking an automorphism which transposes $\alpha_{1}$ with $\overline{\alpha_{1}}$ and fixes $\alpha_{2}$ and $\overline{\alpha_{2}}$, we deduce that $\alpha_{1}=\overline{\alpha_{1}}$, a contradiction.

The only alternative to the above is that each number in the list $L$ occurs at least twice. Note that, without loss of generality, we may assume that $\alpha_{1}$ is non-real and $\alpha_{2} \neq \overline{\alpha_{1}}$. In the case when $\alpha_{4}=\alpha_{1}$ we must have $\alpha_{2}=\overline{\alpha_{2}}$ or $\alpha_{2}=\overline{\alpha_{3}}$. It is clear that $\alpha_{2}=\overline{\alpha_{2}}$ implies that $\alpha_{3}=\overline{\alpha_{3}}$, so both $\alpha_{2}$ and $\alpha_{3}$ are real. Then the lines passing through $\alpha_{1}, \alpha_{2}$ and $\alpha_{3}, \alpha_{4}$ are parallel only if $\alpha_{2}=\alpha_{3}$, a contradiction. Alternatively, if $\alpha_{2}=\overline{\alpha_{3}}$ then $\alpha_{3}=\overline{\alpha_{2}}$, so the numbers $\alpha_{1}, \alpha_{2}, \alpha_{3}, \overline{\alpha_{1}}, \overline{\alpha_{2}}, \overline{\alpha_{3}}$ all must lie on a line parallel to the imaginary axis. We already showed above that this is impossible. Suppose that $\alpha_{4} \neq \alpha_{1}$. Then all four numbers $\alpha_{1}, \alpha_{2}, \alpha_{3}, \alpha_{4}$ are distinct, $\alpha_{1}$ is non-real and $\alpha_{2} \neq \overline{\alpha_{1}}$. It follows that $\alpha_{1} \in\left\{\overline{\alpha_{3}}, \overline{\alpha_{4}}\right\}$. Taking into account the symmetry in the equation $\left(\alpha_{1}-\alpha_{2}\right) /\left(\overline{\alpha_{1}}-\overline{\alpha_{2}}\right)=\left(\alpha_{3}-\alpha_{4}\right) /\left(\overline{\alpha_{3}}-\overline{\alpha_{4}}\right)$, we may assume without loss of generality that $\alpha_{1}=\overline{\alpha_{3}}$. Hence $\alpha_{3}=\overline{\alpha_{1}}$ and the list $L$ becomes $\alpha_{1}, \alpha_{2}, \overline{\alpha_{1}}, \alpha_{4}, \overline{\alpha_{1}}, \overline{\alpha_{2}}, \alpha_{1}, \overline{\alpha_{4}}$. Thus either $\alpha_{2}=\overline{\alpha_{4}}$ or both $\alpha_{2}$ and $\alpha_{4}$ are real (which, in principle, can happen for $d$ even). In the first case, we obtain that $\left(\alpha_{1}-\alpha_{2}\right) /\left(\overline{\alpha_{1}}-\overline{\alpha_{2}}\right)=\left(\overline{\alpha_{1}}-\right.$ $\left.\overline{\alpha_{2}}\right) /\left(\alpha_{1}-\alpha_{2}\right)$, giving $\alpha_{1}-\alpha_{2}= \pm\left(\overline{\alpha_{1}}-\overline{\alpha_{2}}\right)$. Both cases $\alpha_{1}+\overline{\alpha_{1}}=\alpha_{2}+\overline{\alpha_{2}}$ (leading to $\alpha_{1}$ and $\alpha_{2}$ having the same real part) and $\alpha_{1}-\overline{\alpha_{1}}=\alpha_{2}-\overline{\alpha_{2}}$ (see the above paragraph) were already shown to be impossible. If $\alpha_{2}$ and $\alpha_{4}$ are both real then, using $\alpha_{2}=\overline{\alpha_{2}}$ and $\alpha_{4}=\overline{\alpha_{4}}$, we deduce that $\left(\alpha_{1}-\alpha_{2}\right) /\left(\overline{\alpha_{1}}-\alpha_{2}\right)=\left(\overline{\alpha_{1}}-\alpha_{4}\right) /\left(\alpha_{1}-\alpha_{4}\right)$. Subtracting 1 from both sides, we get $\left(\alpha_{1}-\overline{\alpha_{1}}\right) /\left(\overline{\alpha_{1}}-\alpha_{2}\right)=\left(\overline{\alpha_{1}}-\alpha_{1}\right) /\left(\alpha_{1}-\alpha_{4}\right)$. Therefore, $\alpha_{1}+\overline{\alpha_{1}}=\alpha_{2}+\alpha_{4}$. Here, the numbers $\alpha_{1}, \overline{\alpha_{1}}, \alpha_{2}, \alpha_{4}$ are distinct. (For $d=4$, this equality means that all four conjugates form a rhombus.) The Galois group $G$ contains a transposition which interchanges $\alpha_{1}$ with $\alpha_{2}$ and fixes both $\overline{\alpha_{1}}$ and $\alpha_{4}$. Thus $\alpha_{2}+\overline{\alpha_{1}}=\alpha_{1}+\alpha_{4}$, giving $\alpha_{1}=\alpha_{2}$, a contradiction.

\section{No three distinct conjugates of a Salem number lie on a line}

LEMMA 5. Suppose that some three distinct conjugates of a Salem number $\alpha$ lie on a line. Then there exist two distinct conjugates $\alpha^{\prime}$ and $\alpha^{\prime \prime}$ of $\alpha$ such that $\left|\alpha^{\prime}\right|=\left|\alpha^{\prime \prime}\right|=1$ and

$$
\left|\alpha-\alpha^{\prime}\right|\left|\alpha-\alpha^{\prime \prime}\right|=\alpha^{2}-1 \text {. }
$$

Proof. Evidently, three numbers lying on the unit circle $|z|=1$ cannot lie on a line, so either $\alpha$ or $\alpha^{-1}$ (but not both, because these two are the only real conjugates) is among the three conjugates of $\alpha$ lying on a line.

Suppose first that $\alpha, \alpha^{\prime}$ and $\alpha^{\prime \prime}$, where $\left|\alpha^{\prime}\right|=\left|\alpha^{\prime \prime}\right|=1$ and $\alpha^{\prime} \neq \alpha^{\prime \prime}$, lie on a line. Let $A, A^{\prime}$ and $A^{\prime \prime}$ be three points placed at the points of the complex plane $\alpha, \alpha^{\prime}$ and $\alpha^{\prime \prime}$, respectively. Without loss of generality we may assume that $A^{\prime}$ is between $A^{\prime \prime}$ and $A$. Put also $B$ and $B^{\prime}$ 
for 1 and -1 , respectively, so that $B^{\prime}, B$ and $A$ lie on a line. From elementary geometry, by the similarity of triangles $A A^{\prime} B^{\prime}$ and $A A^{\prime \prime} B$, we have $A A^{\prime} \cdot A A^{\prime \prime}=A B^{\prime} \cdot A B$. Hence

$$
\left|\alpha-\alpha^{\prime}\right|\left|\alpha-\alpha^{\prime \prime}\right|=(\alpha+1)(\alpha-1)=\alpha^{2}-1,
$$

as claimed.

Suppose now that $\alpha^{-1}, \alpha_{2}$ and $\alpha_{3}$, where $\left|\alpha_{2}\right|=\left|\alpha_{3}\right|=1$ and $\alpha_{2} \neq \alpha_{3}$, lie on a line. Now, let $A_{1}, A_{2}$ and $A_{3}$ be the points placed at $\alpha^{-1}, \alpha_{2}$ and $\alpha_{3}$, respectively. Clearly, the point $A_{1}$ is between $A_{2}$ and $A_{3}$ and $B^{\prime}, A_{1}$ and $B$ lie on a line. Now, by the similarity of triangles $A_{1} A_{2} B^{\prime}$ and $A_{1} A_{3} B$, we have $A_{1} A_{2} \cdot A_{1} A_{3}=A_{1} B^{\prime} \cdot A_{1} B$ again. Hence

$$
\left|\alpha^{-1}-\alpha_{2}\right|\left|\alpha^{-1}-\alpha_{3}\right|=\left(1+\alpha^{-1}\right)\left(1-\alpha^{-1}\right)=1-\alpha^{-2} .
$$

Set $\alpha^{\prime}=\overline{\alpha_{2}}=\alpha_{2}^{-1}$ and $\alpha^{\prime \prime}=\overline{\alpha_{3}}=\alpha_{3}^{-1}$. Clearly, $\alpha^{\prime}$ and $\alpha^{\prime \prime}$ are conjugate to $\alpha$. Multiplying both sides of the equation $\left|\alpha^{-1}-\alpha^{\prime-1}\right|\left|\alpha^{-1}-\alpha^{\prime \prime-1}\right|=1-\alpha^{-2}$ by $\alpha^{2}=\alpha^{2}\left|\alpha^{\prime}\right|\left|\alpha^{\prime \prime}\right|$ we obtain that

$$
\left|\alpha-\alpha^{\prime}\right|\left|\alpha-\alpha^{\prime \prime}\right|=\alpha^{2}-1
$$

as required.

Set $\beta:=\alpha+\alpha^{-1}$. If $\alpha$ is a Salem number of degree $d$, then $\beta>2$ is a totally real algebraic integer of degree $d / 2$ having its remaining $d / 2-1$ conjugates (different from $\beta$ itself) in the interval $(-2,2)$.

LEMMA 6. Suppose that some three conjugates of a Salem number $\alpha$ lie on a line, and $\beta=\alpha+\alpha^{-1}$. Then there exist two distinct conjugates $\beta^{\prime}$ and $\beta^{\prime \prime}$ of $\beta$ such that

$$
\beta \beta^{\prime}+\beta \beta^{\prime \prime}-\beta^{\prime} \beta^{\prime \prime}=4 .
$$

Proof. Take $\alpha^{\prime}$ and $\alpha^{\prime \prime}$ as in Lemma 5. Set $\beta^{\prime}:=\alpha^{\prime}+\alpha^{-1}$ and $\beta^{\prime \prime}:=\alpha^{\prime \prime}+\alpha^{\prime \prime-1}$. Squaring the equation of Lemma 5 , we find that

$$
\left|\alpha-\alpha^{\prime}\right|^{2}\left|\alpha-\alpha^{\prime \prime}\right|^{2}=\alpha^{4}-2 \alpha^{2}+1=\alpha^{2}\left(\left(\alpha+\alpha^{-1}\right)^{2}-4\right)=\alpha^{2}\left(\beta^{2}-4\right) .
$$

Note that, as $\overline{\alpha^{\prime}}=\alpha^{\prime-1}$,

$$
\left|\alpha-\alpha^{\prime}\right|^{2}=\left(\alpha-\alpha^{\prime}\right)\left(\alpha-\alpha^{\prime-1}\right)=\alpha^{2}-\beta^{\prime} \alpha+1=\alpha\left(\beta-\beta^{\prime}\right) .
$$

Similarly, $\left|\alpha-\alpha^{\prime \prime}\right|^{2}=\alpha\left(\beta-\beta^{\prime \prime}\right)$. Hence $\left(\beta-\beta^{\prime}\right)\left(\beta-\beta^{\prime \prime}\right)=\beta^{2}-4$, giving

$$
4=\beta^{2}-\left(\beta-\beta^{\prime}\right)\left(\beta-\beta^{\prime \prime}\right)=\beta^{2}-\beta^{2}+\beta \beta^{\prime}+\beta \beta^{\prime \prime}-\beta^{\prime} \beta^{\prime \prime}=\beta \beta^{\prime}+\beta \beta^{\prime \prime}-\beta^{\prime} \beta^{\prime \prime},
$$

as claimed.

Suppose that $\beta^{\prime}=\beta^{\prime \prime}$. Then, from $\alpha^{\prime} \neq \alpha^{\prime \prime}$ and $\beta^{\prime}-\beta^{\prime \prime}=\left(\alpha^{\prime}-\alpha^{\prime \prime}\right)\left(1-\left(\alpha^{\prime} \alpha^{\prime \prime}\right)^{-1}\right)$, we deduce that $\alpha^{\prime} \alpha^{\prime \prime}=1$, i.e. $\alpha^{\prime}$ and $\alpha^{\prime \prime}$ are complex conjugate numbers $\alpha^{\prime \prime}=1 / \alpha^{\prime}=\overline{\alpha^{\prime}}$. By Lemma 5, it follows that

$\alpha^{2}-1=\left|\alpha-\alpha^{\prime}\right|\left|\alpha-\alpha^{\prime \prime}\right|=\left(\alpha-\alpha^{\prime}\right)\left(\alpha-\alpha^{\prime-1}\right)=\alpha^{2}-\left(\alpha^{\prime}+\alpha^{\prime-1}\right) \alpha+1=\alpha^{2}-\alpha \beta^{\prime}+1$,

i.e., $\alpha \beta^{\prime}=2$. Hence $\beta^{\prime}=2 / \alpha$. However, the number on the left-hand side is totally real, whereas $2 / \alpha$ has some non-real conjugates, e.g., $2 / \alpha^{\prime}$, a contradiction.

LEMMA 7. Let $u$ and $v$ be two real numbers such that $u+v \neq 0$. Then the three numbers $u, v$ and $(4+u v) /(u+v)$ cannot all belong to the interval $(-2,2)$. 
Proof. Suppose that $u, v \in(-2,2)$. We need to show that $(4+u v) /(u+v)$ is either less than -2 or greater than 2 . Indeed, if $u+v>0$ then $(4+u v) /(u+v)>2$, because $(2-u)(2-v)>0$. If $u+v<0$ then $(2+u)(2+v)>0$ implies the inequality $(4+u v) /$ $(u+v)<-2$.

Proof of Theorem 1. Suppose that some three distinct conjugates of a Salem number $\alpha$ lie on a line. Put $\beta=\alpha+\alpha^{-1}$, and take $\beta^{\prime}, \beta^{\prime \prime}$ as in Lemma 6 .

Recall that $\beta>2$ is an algebraic integer whose conjugates different from $\beta$ itself all lie in the interval $(-2,2)$. Obviously, $\beta^{\prime}+\beta^{\prime \prime} \neq 0$, because $-\beta$ is not a conjugate of $\beta$. So Lemma 6 implies that

$$
\beta=\left(4+\beta^{\prime} \beta^{\prime \prime}\right) /\left(\beta^{\prime}+\beta^{\prime \prime}\right) .
$$

Applying an automorphism $\sigma$ that maps $\beta \mapsto \beta^{\prime}$, we obtain $\beta^{\prime}=\left(4+\sigma\left(\beta^{\prime}\right) \sigma\left(\beta^{\prime \prime}\right)\right) /\left(\sigma\left(\beta^{\prime}\right)+\right.$ $\sigma\left(\beta^{\prime \prime}\right)$ ), where, by Lemma 7, either $\sigma\left(\beta^{\prime}\right)$ or $\sigma\left(\beta^{\prime \prime}\right)$ must be $\beta$. Denoting by $\beta^{*}$ the other conjugate, so that $\left\{\sigma\left(\beta^{\prime}\right), \sigma\left(\beta^{\prime \prime}\right)\right\}=\left\{\beta, \beta^{*}\right\}$, we have

$$
\beta^{\prime}=\left(4+\beta \beta^{*}\right) /\left(\beta+\beta^{*}\right) .
$$

Subtracting $\beta^{\prime}\left(\beta+\beta^{*}\right)=4+\beta \beta^{*}$ from $\beta\left(\beta^{\prime}+\beta^{\prime \prime}\right)=4+\beta^{\prime} \beta^{\prime \prime}$, we obtain $\beta \beta^{\prime \prime}-\beta^{\prime} \beta^{*}=$ $\beta^{\prime} \beta^{\prime \prime}-\beta \beta^{*}$. Hence $\left(\beta-\beta^{\prime}\right)\left(\beta^{\prime \prime}+\beta^{*}\right)=0$, which is impossible, because $\beta \neq \beta^{\prime}$ and, as above, $\beta^{\prime \prime}+\beta^{*} \neq 0$.

\section{Proof of Theorem 2}

Let $\alpha$ be a Salem number of degree $d$. We shall denote its distinct conjugates on the unit circle by $\alpha_{1}, \alpha_{2}, \ldots$, frequently using the fact that $\overline{\alpha_{j}}=\alpha_{j}^{-1}$. We suppose that there exist two pairs of conjugates such that the lines passing through each pair are parallel. There are eight possibilities for these two pairs:

(a) $\alpha_{1}, \alpha_{2}$ and $\alpha_{3}, \alpha_{4}$;

(b) $\alpha, \alpha^{-1}$ and $\alpha_{1}, \alpha_{2}$;

(c) $\alpha, \alpha_{1}$ and $\alpha_{2}, \alpha_{3}$, where $\alpha_{1} \notin\left\{\alpha_{2}^{-1}, \alpha_{3}^{-1}\right\}$;

(c) $\alpha^{-1}, \alpha_{1}$ and $\alpha_{2}, \alpha_{3}$, where $\alpha_{1} \notin\left\{\alpha_{2}^{-1}, \alpha_{3}^{-1}\right\}$;

(d) $\alpha, \alpha_{1}$ and $\alpha_{2}, \alpha_{1}^{-1}$;

(d') $\alpha^{-1}, \alpha_{1}$ and $\alpha_{2}, \alpha_{1}^{-1}$;

(e) $\alpha, \alpha_{1}$ and $\alpha^{-1}, \alpha_{1}^{-1}$;

(f) $\alpha, \alpha_{1}$ and $\alpha^{-1}, \alpha_{2}$, where $\alpha_{2} \neq \alpha_{1}^{-1}$.

We begin with case $(a)$. The line through $\alpha_{1}, \alpha_{2}$ is parallel to the line through $\alpha_{3}, \alpha_{4}$ if there is a real constant $k$ such that $\alpha_{1}-\alpha_{2}=k\left(\alpha_{3}-\alpha_{4}\right)$. Taking complex conjugates, we have $\overline{\alpha_{1}}-\overline{\alpha_{2}}=k\left(\overline{\alpha_{3}}-\overline{\alpha_{4}}\right)$ and, using $\overline{\alpha_{i}}=\alpha_{i}^{-1}$, we obtain $\left(\alpha_{2}-\alpha_{1}\right) / \alpha_{1} \alpha_{2}=k\left(\alpha_{4}-\alpha_{3}\right) / \alpha_{3} \alpha_{4}$. Hence $\alpha_{1} \alpha_{2}=\alpha_{3} \alpha_{4}$. By Salem's result, this is only possible if $\left\{\alpha_{1}, \alpha_{2}\right\}=\left\{\alpha_{3}, \alpha_{4}\right\}$, a contradiction.

In case $(b)$ the line passing through $\alpha_{1}$ and $\alpha_{2}$ must be parallel to the real axis, so $\alpha_{1} \alpha_{2}=-1$. This is impossible, by Salem's result.

In case (c) we have $\alpha-\alpha_{1}=k\left(\alpha_{2}-\alpha_{3}\right)$ for some real constant $k$. Thus $\alpha-\overline{\alpha_{1}}=k\left(\overline{\alpha_{2}}-\overline{\alpha_{3}}\right)$, which is equivalent to $\alpha-\alpha_{1}^{-1}=k\left(\alpha_{3}-\alpha_{2}\right) / \alpha_{2} \alpha_{3}$. Thus $\alpha-\alpha_{1}=-\left(\alpha-\alpha_{1}^{-1}\right) \alpha_{2} \alpha_{3}$. Map $\alpha_{2}$ to $\alpha$. Then, as $\alpha_{2}^{-1} \mapsto \alpha^{-1}$ and $\alpha_{2}^{-1} \notin\left\{\alpha, \alpha_{1}, \alpha_{3}\right\}$, we see that $\alpha \mapsto \alpha_{4}, \alpha_{1} \mapsto \alpha_{5}$ and $\alpha_{3} \mapsto$ $\alpha_{6}$. (In principle, $\alpha_{4}, \alpha_{5}, \alpha_{6}$ can be $\alpha_{2}$ or $\alpha_{3}$.) We thus obtain $\alpha_{4}-\alpha_{5}=-\left(\alpha_{4}-\alpha_{5}^{-1}\right) \alpha \alpha_{6}$, i.e., $\alpha_{4}-\alpha_{5}=\alpha\left(\alpha_{6} / \alpha_{5}\right)\left(1-\alpha_{4} \alpha_{5}\right)$. Now, consider the corresponding complex conjugate equation $\overline{\alpha_{4}}-\overline{\alpha_{5}}=\alpha\left(\overline{\alpha_{6}} / \overline{\alpha_{5}}\right)\left(1-\overline{\alpha_{4} \alpha_{5}}\right)$, equivalent to $\alpha_{4}-\alpha_{5}=\alpha\left(\alpha_{5} / \alpha_{6}\right)\left(1-\alpha_{4} \alpha_{5}\right)$. This gives $\alpha_{5} / \alpha_{6}=\alpha_{6} / \alpha_{5}$, i.e., $\alpha_{5}^{2}=\alpha_{6}^{2}$, contradicting Salem's result again. 
Case $\left(c^{\prime}\right)$ can be worked out in the same manner as (c) with $\alpha$ replaced by $\alpha^{-1}$. So we now turn to case $(d)$. Then, as above, there is a real number $k$ such that $\alpha-\alpha_{1}=k\left(\alpha_{2}-\alpha_{1}^{-1}\right)$. Taking complex conjugates, we obtain $\alpha-\alpha_{1}^{-1}=k\left(\alpha_{2}^{-1}-\alpha_{1}\right)$. Dividing one equation by the other, we get $\left(\alpha-\alpha_{1}\right) /\left(\alpha-\alpha_{1}^{-1}\right)=-\alpha_{2} / \alpha_{1}$, so $\left(\alpha-\alpha_{1}\right) \alpha_{1}+\left(\alpha-\alpha_{1}^{-1}\right) \alpha_{2}=0$. Multiplying by $\alpha_{1}$ we get $\alpha \alpha_{1}\left(\alpha_{1}+\alpha_{2}\right)=\alpha_{1}^{3}+\alpha_{2}$. Map $\alpha_{2}$ to $\alpha$. Suppose that $\alpha \mapsto \alpha_{3}$ and $\alpha_{1} \mapsto \alpha_{4}$. (Here, $\alpha_{3}, \alpha_{4}$ can be $\alpha_{1}$ or $\alpha_{2}$.) It follows that $\alpha_{3} \alpha_{4}\left(\alpha+\alpha_{4}\right)=\alpha_{4}^{3}+\alpha$, i.e., $\alpha\left(1-\alpha_{3} \alpha_{4}\right)=\alpha_{4}^{2}\left(\alpha_{3}-\alpha_{4}\right)$. Taking the complex conjugate equation we get $\alpha\left(\alpha_{3} \alpha_{4}-1\right) / \alpha_{3} \alpha_{4}=\left(\alpha_{4}-\alpha_{3}\right) / \alpha_{3} \alpha_{4}^{3}$, i.e., $\alpha\left(1-\alpha_{3} \alpha_{4}\right)=\alpha_{4}^{-2}\left(\alpha_{3}-\alpha_{4}\right)$. Thus $\alpha_{4}^{2}=\alpha_{4}^{-2}$, giving $\alpha_{4}^{4}=1$, a contradiction to Salem's result.

The case $\left(d^{\prime}\right)$ is essentially the same as $(d)$. (One only needs to replace $\alpha$ by $\alpha^{-1}$ in the above argument.) In case (e) we have $\alpha-\alpha_{1}=k\left(\alpha^{-1}-\alpha_{1}^{-1}\right)=k\left(\alpha_{1}-\alpha\right)\left(\alpha \alpha_{1}\right)^{-1}$ with some real number $k$. Thus $\alpha \alpha_{1}=-k$, which is impossible, because the left-hand side is non-real.

Finally, in case $(f)$ we have $\alpha-\alpha_{1}=k\left(\alpha^{-1}-\alpha_{2}\right)$. Clearly, $\alpha_{1} \notin\left\{\alpha_{2}, \alpha_{2}^{-1}\right\}$. Dividing this equation by its complex conjugate $\alpha-\alpha_{1}^{-1}=k\left(\alpha^{-1}-\alpha_{2}^{-1}\right)$ we obtain $\left(\alpha-\alpha_{1}\right) /(\alpha-$ $\left.\alpha_{1}^{-1}\right)=\left(\alpha^{-1}-\alpha_{2}\right) /\left(\alpha^{-1}-\alpha_{2}^{-1}\right)$. The right-hand side is equal to $\alpha_{2}\left(1-\alpha \alpha_{2}\right) /\left(\alpha_{2}-\alpha\right)$. So $\left(\alpha-\alpha_{1}\right)\left(\alpha_{2}-\alpha\right)=\left(\alpha-\alpha_{1}^{-1}\right)\left(1-\alpha \alpha_{2}\right) \alpha_{2}$. It follows that

$$
\alpha^{2} \alpha_{1}\left(\alpha_{2}^{2}-1\right)+\alpha\left(\alpha_{1}^{2}-\alpha_{2}^{2}\right)-\left(\alpha_{1}^{2}-1\right) \alpha_{2}=0 .
$$

Next, map $\alpha_{1} \mapsto \alpha$. Suppose that this automorphism maps $\alpha \mapsto \alpha_{3}$ and $\alpha_{2} \mapsto \alpha_{4}$. (Once again, $\alpha_{3}, \alpha_{4}$ and $\alpha_{5}, \alpha_{6}$, which we introduce below, can be $\alpha_{1}$ or $\alpha_{2}$.) We obtain that $\alpha_{3}^{2} \alpha\left(\alpha_{4}^{2}-1\right)+\alpha_{3}\left(\alpha^{2}-\alpha_{4}^{2}\right)-\left(\alpha^{2}-1\right) \alpha_{4}=0$, i.e.,

$$
\alpha^{2}\left(\alpha_{3}-\alpha_{4}\right)+\alpha \alpha_{3}^{2}\left(\alpha_{4}^{2}-1\right)+\alpha_{4}\left(1-\alpha_{3} \alpha_{4}\right)=0 .
$$

By taking the complex conjugate equation we deduce that

$$
-\alpha^{2}\left(\alpha_{3}-\alpha_{4}\right)+\alpha \alpha_{3}^{-1}\left(\alpha_{4}^{-1}-\alpha_{4}\right)+\alpha_{3}-\alpha_{4}^{-1}=0 .
$$

Adding these two equations yields

$$
\alpha\left(\alpha_{4}^{2}-1\right)\left(\alpha_{3}^{2}-\left(\alpha_{3} \alpha_{4}\right)^{-1}\right)+\alpha_{4}-\alpha_{3} \alpha_{4}^{2}+\alpha_{3}-\alpha_{4}^{-1}=0 .
$$

This is equivalent to $\left(\alpha_{4}^{2}-1\right)\left(\alpha_{3} \alpha_{4}\right)^{-1}\left(\alpha\left(\alpha_{3}^{3} \alpha_{4}-1\right)+\alpha_{3}\left(1-\alpha_{3} \alpha_{4}\right)\right)=0$. Since $\alpha_{4}^{2} \neq 1$, we obtain that $\alpha\left(\alpha_{3}^{3} \alpha_{4}-1\right)+\alpha_{3}\left(1-\alpha_{3} \alpha_{4}\right)=0$. Hence $\alpha_{4} \alpha_{3}^{2}\left(\alpha \alpha_{3}-1\right)=\alpha-\alpha_{3}$. Map $\alpha_{4} \mapsto \alpha$. Suppose that then $\alpha \mapsto \alpha_{5}$ and $\alpha_{3} \mapsto \alpha_{6}$. This automorphism maps the last equality into $\alpha \alpha_{6}^{2}\left(\alpha_{5} \alpha_{6}-1\right)=\alpha_{5}-\alpha_{6}$. The corresponding complex conjugate equation is $\alpha \alpha_{6}^{-2}\left(\left(\alpha_{5} \alpha_{6}\right)^{-1}-\right.$ $1)=\alpha_{5}^{-1}-\alpha_{6}^{-1}$. Multiplying by $-\alpha_{5} \alpha_{6}$, we obtain $\alpha \alpha_{6}^{-2}\left(\alpha_{5} \alpha_{6}-1\right)=\alpha_{5}-\alpha_{6}$. Thus $\alpha_{6}^{2}=\alpha_{6}^{-2}$, giving $\alpha_{6}^{4}=1$, a contradiction. This completes the proof of the theorem.

\section{Pisot numbers}

Pisot numbers are close relatives of Salem numbers. Recall that a Pisot number $\alpha>1$ is an algebraic integer whose conjugates, except for $\alpha$ itself, all lie in the unit disc $|z|<1$. It is known that at most two conjugates of a Pisot number can have the same modulus [3]. Moreover, Mignotte [1] proved that there are no non-trivial multiplicative relations between the conjugates of a Pisot number. This implies, for instance, that no two non-real conjugates of a Pisot number can have the same argument. Otherwise, we would obtain the multiplicative relation $\alpha_{1} \alpha_{2}=\alpha_{3} \alpha_{4}$ in conjugates of a Pisot number, which, by Mignotte's result, is impossible.

(i) Can two non-real conjugates of a Pisot number have the same imaginary part?

(ii) Can four conjugates of a Pisot number have the same real part? 
Note that it cannot be the case that exactly three conjugates of a Pisot number have the same real part. Indeed, if so, then one of these three, say, $\alpha_{1}$ must be real and the other two, say, $\alpha_{2}, \alpha_{3}$ must be complex conjugates, i.e., $\alpha_{3}=\overline{\alpha_{2}}$. However, this leads to the equation $2 \alpha_{1}=\alpha_{2}+\alpha_{3}$ which, by [4, lemma 1], has no solutions in distinct conjugates of an algebraic number.

Both questions lead easily to the following non-trivial additive relation

$$
\alpha_{1}+\alpha_{2}=\alpha_{3}+\alpha_{4}
$$

in distinct conjugates of a Pisot number. It is quite tempting to conjecture that, similarly to the multiplicative case, this equation has no solutions in such conjugates. If so, this would imply that the answer to both questions posed above is negative. However, the situation is not that simple, as this equation is solvable in conjugates of a Pisot number. We give the following example.

Set $\alpha:=(1+\sqrt{3+2 \sqrt{5}}) / 2$. The minimal polynomial of $\alpha$ is $x^{4}-2 x^{3}+x-1$. It is a Pisot number, with conjugates $\alpha_{2}=(1-\sqrt{3+2 \sqrt{5}}) / 2, \alpha_{3}=(1+i \sqrt{-3+2 \sqrt{5}}) / 2$ and $\alpha_{4}=(1-$ $i \sqrt{-3+2 \sqrt{5}}) / 2$. Here, $\alpha=1.86676 \ldots, \alpha_{2}=-0.86676 \ldots,\left|\alpha_{3}\right|=\left|\alpha_{4}\right|=0.78615 \ldots$ Hence two real numbers $\alpha, \alpha_{2}$ and two complex conjugate numbers $\alpha_{3}$ and $\alpha_{4}$ satisfy

$$
\alpha+\alpha_{2}=\alpha_{3}+\alpha_{4}=1 \text {. }
$$

It follows that the numbers $\alpha, \alpha_{3}, \alpha_{2}, \alpha_{4}$ form a rhombus in the complex plane.

The minimal polynomial $x^{4}-2 x^{3}+x-1$ of the $\alpha$ just given can be written in the form $P\left(x^{2}-x\right)$, where $P(z)=z^{2}-z-1$. This kind of construction gives other, albeit non-Pisot, examples of interest. For instance, when $P(z)=z^{3}-z^{2}-1$ the polynomial $P\left(x^{2}-x\right)=x^{6}-3 x^{5}+2 x^{4}+x^{3}-x^{2}-1$ is irreducible and has two roots with the same imaginary part. However, it has three roots outside the unit circle, so does not give a Pisot number.

When $P(z)=z^{3}+4 z^{2}+3 z-1$ the polynomial $P\left(x^{2}-x\right)=x^{6}-3 x^{5}+7 x^{4}-9 x^{3}+$ $7 x^{2}-3 x-1$ is again irreducible, and has four roots with the same real part. But it has five roots outside the unit circle.

We end with a speculative construction method which just may be able to answer these questions in the affirmative. For this, we need a simple result.

Lemma 8. Let $\beta, \gamma \in \mathbb{C}$. Then the polynomial $z^{2}-\beta z-\gamma$ has:

(i) two roots in $|z|<1$ when $|\gamma|<1$ and $|\beta|<|\gamma-1|$;

(ii) exactly one root in $|z|<1$ when $|\gamma| \neq 1$ and $|\beta|>|\gamma-1|$;

(iii) no roots in $|z|<1$ when $|\gamma|>1$ and $|\beta|<|\gamma-1|$.

Now suppose that $\beta$ is real. Then:

(iv) if $\gamma$ is non-real, then $\left(z^{2}-\beta z-\gamma\right)\left(z^{2}-\beta z-\bar{\gamma}\right)$ has two non-real roots with the same imaginary part;

(v) if $\gamma$ and $\gamma^{\prime}$ are both real and less than $-\beta^{2} / 4$, then all four roots of $\left(z^{2}-\beta z-\right.$ $\gamma)\left(z^{2}-\beta z-\gamma^{\prime}\right)$ have the same real part.

The first part of the proof comes from applying Rouché's Theorem to the identity

$$
\left(\gamma z^{2}+\beta z-1\right)-\gamma\left(z^{2}-\beta z-\gamma\right)=(1+\gamma)(\beta z+\gamma-1) .
$$

The second part is an easy exercise. 
We now indicate how the lemma might be applied. Suppose that $\beta$ is algebraic with $\gamma$ of degree at least 2 over $\mathbb{Q}(\beta)$, and that $x^{2}-\beta x-\gamma$ is irreducible over $\mathbb{Q}(\gamma)$. For simplicity of exposition, we shall assume from now on that in fact $\gamma$ is quadratic over $\mathbb{Q}(\beta)$ with $\gamma^{\prime}$ being its conjugate. Suppose that $d \geqslant 2, \beta$ has conjugates $\beta=\beta_{1}, \ldots, \beta_{d}$ and that the conjugates of $\gamma$ that are quadratic over $\mathbb{Q}\left(\beta_{i}\right)$ are $\gamma_{i}$ and $\gamma_{i}^{\prime}$. Suppose too that $\left|\gamma_{1}\right|>1,\left|\gamma_{i}\right|<1 \quad(i=$ $2, \ldots, d),\left|\gamma_{i}^{\prime}\right|<1 \quad(i=1, \ldots, d),\left|\gamma_{1}-1\right|<\left|\beta_{1}\right|<\left|\gamma_{1}^{\prime}-1\right|$ while $\left|\beta_{i}\right|<\min \left(\left|\gamma_{i}-1\right|, \mid \gamma_{i}^{\prime}-\right.$ 1|) for $i=2, \ldots, d$. Then

$$
Q(x):=\prod_{i=1}^{d}\left(x^{2}-\beta_{i} x-\gamma_{i}\right)\left(x^{2}-\beta_{i} x-\gamma_{i}^{\prime}\right) \in \mathbb{Q}[x]
$$

has one root of modulus greater than 1 and the rest of modulus less than 1 . It is therefore irreducible and so, for $\beta$ and $\gamma$ algebraic integers, $Q$ is the minimal polynomial of \pm a Pisot number. Further:

(i) if $\beta_{2}$ (say) is real and $\gamma_{2}$ non-real, then, by Lemma 8 , we would obtain a Pisot number with two non-real conjugates having the same imaginary part;

(ii) if $\beta_{2}$ is real and $\gamma_{2}$ and $\gamma_{2}^{\prime}$ are both real and less than $-\beta_{2}^{2} / 4$, then, by Lemma 8 , we would obtain a Pisot number with four conjugates having the same real part.

On the other hand, we have been able neither to make use of Lemma 8 nor to find another example of a Pisot number $\alpha$ whose conjugates satisfy $\alpha_{1}+\alpha_{2}=\alpha_{3}+\alpha_{4}$, except for $\alpha=(1+$ $\sqrt{3+2 \sqrt{5}}$ ) $/ 2$. If one were able to show that there are only finitely many such examples, it would be a step towards answering our questions in the negative. The most we can say is that such an $\alpha$ must be less than 3: this comes immediately from mapping $\alpha_{1}$ in $\alpha_{1}=\alpha_{3}+\alpha_{4}-\alpha_{2}$ to $\alpha$ by an appropriate automorphism.

Acknowledgements. We thank a referee for some useful corrections. The research of the first named author was supported in part by the Lithuanian State Studies and Science Foundation.

\section{REFERENCES}

[1] M. Mignotte. Sur les conjugées des nombres de Pisot. C. R. Acad. Sci. Paris Sér. I. Math. 298 (1984), 21.

[2] J.-P. SERRE. Topics in Galois Theory. Research Notes in Mathematics 1 (Jones \& Bartlett, 1992).

[3] C. J. SмYTH. The conjugates of algebraic integers. Amer. Math. Monthly 82 (1975), 86.

[4] C. J. Sмyтh. Conjugate algebraic numbers on conics. Acta Arith. 40 (1982), 333-346.

[5] R. SALEM. Algebraic Numbers and Fourier Analysis (D. C. Heath, 1963). 